\title{
The energy spectra of the graphene-based quasi-periodic superlattice
}

\author{
A.M. Korol ${ }^{1,2}$, A.I. Sokolenko ${ }^{2}$, and I.V. Sokolenko ${ }^{3}$ \\ ${ }^{1}$ Laboratory on Quantum Theory in Linkoping, ISIR, P.O. Box 8017, S-580, Linkoping, Sweden \\ ${ }^{2}$ National University for Food Technologies, 68, Volodymyrska str., Kyiv 01601, Ukraine \\ E-mail: korolam@ukr.net \\ ${ }^{3}$ Krypton Ocean Group, 37/97, Zhylians'ka str., Kyiv 01033, Ukraine
}

Received January 1, 2018, revised March 15, 2018, published online June 27, 2018

\begin{abstract}
The spectra of the Dirac quasi-electrons transmission through the Fibonacci quasi-periodical superlattice (SL) are calculated and analyzed in the continuum model with the help of the transfer matrix method. The onedimensional SL based on a monolayer graphene modulated by the Fermi velocity barriers is studied. A new quasi-periodical factor is proposed to be considered. We show that the Fibonacci quasi-periodic modulation in graphene superlattices with the velocity barriers can be effectively realized by virtue of a difference in the velocity barrier values (no additional factor is needed and we keep in mind that not each factor can provide the quasi-periodicity). This fact is true for a case of normal incidence of quasi-electrons on a lattice. In contrast to the case of other types of the graphene SL spectra studied reveal the remarkable property, namely the periodic character over all the energy scale and the transmission coefficient doesn't tend asymptotically to unity at rather large energies. Both the conductance (using the known Landauer-Buttiker formula) and the Fano factor for the structure considered are also calculated and analyzed. The dependence of spectra on the Fermi velocity magnitude and on the external electrostatic potential as well as on the SL geometrical parameters (width of barriers and quantum wells) is analyzed. Using the quasi-periodical SL one can control the transport properties of the graphene structures in a wide range. The obtained results can be used for applications in the graphene-based electronics.
\end{abstract}

PACS: 73.21.Cd Superlattices;

73.63.-b Electron transport in nanomaterials and structures.

Keywords: graphene, Fibonacci superlattice, velocity barriers, transmission spectra.

\section{Introduction}

Graphene and the graphene-based structures draw the great attention of researchers in recent years. It is explained by the unique physical properties of graphene, and also by good prospects of its use in the nanoelectronics (see, e.g., [1-4]). It is convenient to control the behavior of the Weyl-Dirac fermions in graphene by means of the external electric and magnetic fields, and a lot of publications are devoted to the corresponding problem for this reason. Recently one more way of controlling the electronic properties of the graphene structures, namely by means of the spatial change of the Fermi velocity was offered [5-10]. Some ways of fabrication of structures in which the Fermi velocity of quasi-particles is spatially dependent value were approved $[5,6]$. This achievement in the technology opens new opportunities for receiving the nanoelectronic devices with the desirable transport properties.
It is known that the solution of this problem can be promoted to the great extent by use of the superlattices. This explains the emergence of a number of publications in which the charge carriers behavior in graphene superlattices of various types is investigated; these SL include the strictly periodic, the disordered ones, SL with barriers of various nature - electrostatic, magnetic, barriers of Fermi velocity (under which we understand the areas of graphene where quasi-particles have different Fermi velocity, smaller or bigger than in the pristine graphene). In particular, in recent papers [7] and especially [11] the influence of the Fermi velocity barriers on the electronic properties of the strictly periodic graphene superlattices was analyzed. It was shown that it is possible to tune the transmission rates from zero to unity only changing the Fermi velocity, also to control the energy gap value and the amplitudes and location of the resonant peaks in the conductivity. 
Among the specified works, there are some devoted to the quasi-periodic graphene SL [12-16]. The quasi-periodic structures, as known, possess the unusual electronic properties of special interest (see, e.g., [17]), such as self-similarity, the Cantor nature of the energy spectrum, etc.

Motivated by the circumstances stated above we formulate the purpose of this work as follows: to study the main features of the transmission spectra of the quasi-periodical graphene-based Fibonacci superlattices with the velocity barriers. We show that using the quasi-periodic superlattices gives the additional possibilities to control the transport properties of the graphene-based structures flexibly. We choose the Fibonacci SL because they are considered as the classical quasi-periodic objects, and the majority of the works associated with research of the quasi-periodic systems deal merely with them.

\section{Model and formulae}

Consider the one-dimensional graphene superlattice in which regions with various values of the Fermi velocity are located along the $0 x$ axis: elements $a$ and $b$ refer to $v_{a}$ and $v_{b}$ velocities, respectively. Elements $a$ and $b$ are arranged along SL according to the Fibonacci rule so that, for example, we have for the fourth Fibonacci generation (sequence): $s_{4}=a b a a b$. Generally, between the barriers corresponding to elements $a$ and $b$, there is a quantum well for which the Fermi velocity is equal to unity as in a pristine graphene: $v_{w}=v_{0}$.

As we consider graphene in which the Fermi velocity is dependent on the spatial coordinate $\mathbf{r}$, i.e., $v=v(\mathbf{r})$ the quasi-particles submit to the massless Weyl-Dirac type equation:

$$
-i \hbar \boldsymbol{\sigma} \cdot \nabla[\sqrt{\mathbf{v}(\mathbf{r})} \phi(\mathbf{r})] \sqrt{\mathbf{v}(\mathbf{r})}=E \phi(\mathbf{r})
$$

where $\boldsymbol{\sigma}=\left(\sigma_{x} ; \sigma_{y}\right)$ is the Pauli two-dimensional matrix; $\phi(\mathbf{r})=\left[\phi_{A}(\mathbf{r}) ; \phi_{B}(\mathbf{r})\right] T$ is the two-component spinor, $T$ is the transposing symbol. Introducing an auxiliary spinor $\Phi(\mathbf{r})=\sqrt{\mathbf{v}(\mathbf{r})} \phi(\mathbf{r})$ one can rewrite equation (1) as follows:

$$
-i \hbar \mathbf{v}(\mathbf{r}) \boldsymbol{\sigma} \cdot \nabla \Phi(\mathbf{r})=E \Phi(\mathbf{r})
$$

Assume that the external potential consists of the periodically repeating rectangular velocity barriers along the axis $0 x$ and potential is constant in each $j$ th barrier. The external electrostatic potential $U$ may also be present and inside each barrier $U_{j}(x)=$ const (piece-wise constant potential). In this case using the translational invariance of the solution over the $0 y$ axis it is possible to receive from the Eq. (2):

$$
\frac{d^{2} \Phi_{A, B}}{d x^{2}}+\left(k_{j}^{2}-k_{y}^{2}\right) \Phi_{A, B}=0,
$$

where indices $A, B$ relate to the graphene sublattices $A$ and $B$, respectively, $k_{j}=\left[E-U_{j}(x)\right] / v_{j}$, measurement units $\hbar=v_{0}=1$ are accepted. If we represent the solution for eigenfunctions in the form of the plane waves moving in the direct and opposite direction along an axis $0 x$, we derive

$$
\Phi(x)=\left[a_{j} \mathrm{e}^{i q_{j} x}\left(\begin{array}{c}
1 \\
g_{j}^{+}
\end{array}\right)+b_{j} \mathrm{e}^{-i q_{j} x}\left(\begin{array}{c}
1 \\
g_{j}^{-}
\end{array}\right)\right],
$$

where $q_{j}=\sqrt{k_{j}^{2}-k_{y}^{2}}$ for $k_{j}^{2}>k_{y}^{2}$ and $q_{j}=i \sqrt{k_{y}^{2}-k_{j}^{2}}$ otherwise, $g_{j}^{ \pm}=\left( \pm q_{j}+i k_{y}\right) v_{j} /\left(E-U_{j}\right)$, the top line in (4) pertains to the sublattice $A$, the lower one - to the sublattice $B$.

The transfer matrix which associates wave functions in points $x$ and $x+\Delta x$ reads

$$
M_{j}=\frac{1}{\cos \theta_{j}}\left(\begin{array}{cc}
\cos \left(q_{j} \Delta x-\theta_{j}\right) & i \sin \left(q_{j} \Delta x\right) \\
i \sin \left(q_{j} \Delta x\right) & \cos \left(q_{j} \Delta x+\theta_{j}\right)
\end{array}\right),
$$

where $\theta_{j}=\arcsin \left(k_{y} / k_{j}\right)$.

Meaning that the Fermi velocity depends only on coordinate $x$, i.e. $v(\mathbf{r})=v(x)$, it is possible to receive the boundary matching condition from the continuity equation for the current density as follows:

$$
\sqrt{v_{b}} \phi\left(x_{b w}^{-}\right)=\sqrt{v_{w}} \phi\left(x_{b w}^{+}\right)
$$

where indexes $b$ and $w$ relate to a barrier and a quantum well, respectively, $x_{b w}$ is the coordinate of the barrier-well interface. The coefficient of transmission of quasi-electrons through the superlattice $T(E)$ is evaluated by means of a transfer matrix method and it is equal to $T=|t|^{2}$,

$$
t=\frac{2 \cos \theta_{0}}{R_{22} \mathrm{e}^{-i \theta_{0}}+R_{11} \mathrm{e}^{i \theta_{0}}-R_{12}-R_{21}},
$$

where $\theta_{0}$ is the angle of incidence of the quasi-particles on the lattice and the matrix $R$ is expressed via the product of the matrices $M_{j}: R=\prod_{j=1}^{N} M_{j}, N$ is the total number of elements in the SL. Energy ranges for which the coefficient of electron transmission through the lattice is close to unity form the allowed bands while the energy gaps correspond to values $T<<1$. Now we have an opportunity to proceed with analyzing the obtained results.

\section{Results and discussion}

Unlike the energy spectra for the known quasi-periodic superlattices, including the graphene ones (see, e.g., $[7,15,16])$, the spectra of the graphene-based SL with the velocity barriers are periodic over all the energy scale, and the transmission rate $T$ doesn't tend asymptotically to unity at rather large energies. For comparison, dependences of $\log T(E)$ are given in Fig. 1(a) for the Fibonacci fourth 

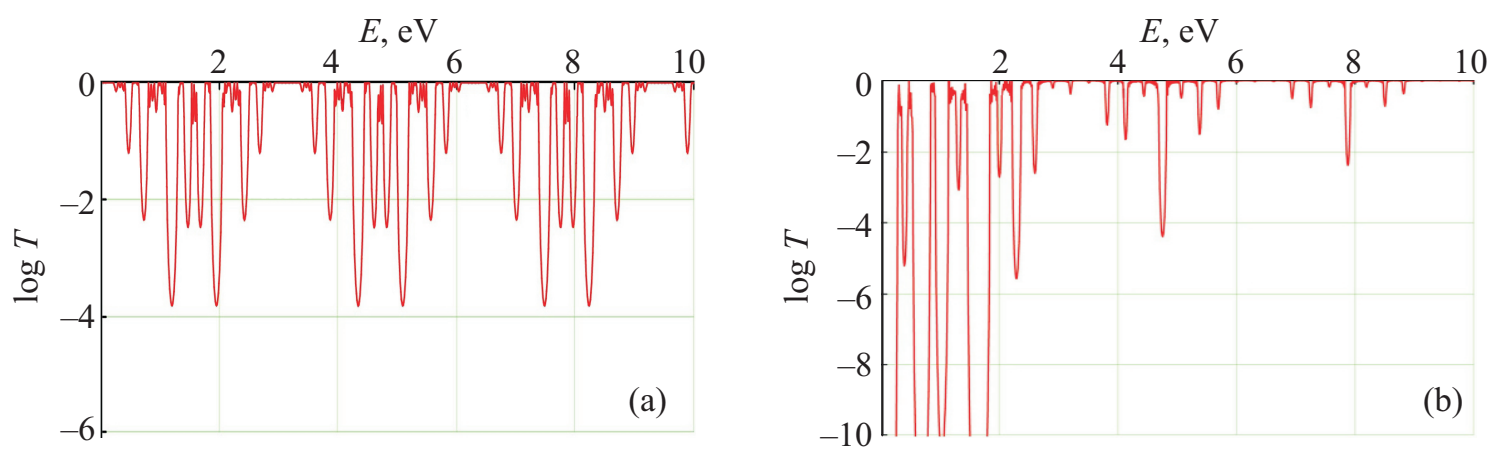

Fig. 1. Dependences of $\log T$ on energy $E$ for the SL modulated by: (a) different values of the Fermi velocity and (b) different magnitudes of the energy gaps.

generation for SL in which the quasi-periodic modulation is achieved due to different values of the Fermi velocity, and for SL on the basis of the gapped graphene in which the quasi-periodic modulation is due to different values of gaps (calculations are carried out on the basis of our previous work [15], Fig. 1(b)). The values of the parameters are as follows: for the first case $w=10 \mathrm{~nm}, d=20 \mathrm{~nm}, v_{a}=1$, $v_{b}=2$, for the second case $w=d=10 \mathrm{~nm}, \Delta_{a}=0.08 \mathrm{eV}$, $\Delta_{b}=0$, where $\Delta$ denotes the gap's width, $d$ and $w$ denotes the barrier and the quantum well width, respectively. All calculations (for all figures of this paper) were carried out for the case of the normal incidence of electrons on the superlattice. (Note that in accordance with the known Landauer-Buttiker formula the electrons with $k_{y}=0$ make the main contribution to the conductance).

It is seen that a certain periodicity of spectra takes place in the second case (this fact hasn't been noted in the literature as yet) but the amplitude of peaks (and the corresponding gap's width) decreases with increasing in $E$, on average. The allowed band width increases on average with $E$ increasing and the coefficient of transmission $T$ eventually approaches to unity. This "wavy damped oscillation” in Fig. 1(b) is associated with such property of the spectra as their self-similarity (e.g., [15]). Note that the narrowing of gaps occurs very rapidly. Parameters for the spectra in Fig. 1 are chosen so as to show that their structure for the graphene SL of different nature may be similar. The difference of two spectra is explained by that the velocity barriers are dependent on energy [9]. If we make an analogy between tunneling of quasi-particles in graphene through a rectangular electrostatic barrier and tunneling through a velocity barrier, for the potential of the last it is necessary to write down

$$
U(E)=E-E / v_{b},
$$

in other words expressions for the transmission coefficient $T$ in the specified cases coincide if the condition (7) is satisfied. This formula explains the fact that spectra of $T(E)$ for SL with the velocity barriers are periodic over all the energy scale. It is quite naturally that the expression for the transmission rates comprises the term that directly deter- mines the spectra periodicity (see, e.g., the recent papers $[7,19,20])$.

Note further that the graphene superlattices with the velocity barriers are characterized by a rich variety of the energy spectra, and also by their high sensitivity to minor changes in geometrical parameters of a lattice. This statement is correct in relation not only to quasi-periodically modulated SL, but to strictly periodic lattices as well and it allows for controlling the energy spectra in a wide range. In the general case, i.e., for arbitrary values of the parameter values, the energy spectra demonstrate a set of irregularly spaced of allowed and forbidden bands. However for some sets of the parameter values spectra are regular and it is natural to take them for analysis in the first place; examples of such spectra are shown in figures of this paper. (The same conclusion in relation to the strictly periodic SL with the velocity barriers was done in [19,20].)

Apparently, depending on the parameters of the problem under consideration spectra may differ from each other significantly; they can reveal the simple form with the small minimal period equal to several energy units, but also they can expose much more complicated pattern of bands with the minimal period of several tens of energy units. Each set of values of parameters provides the original specter with its own minimal period and substructure. In the minimal period of each specter, there is a point with respect to which the specter is symmetric and besides each specter exhibits a symmetric substructure (e.g., Fig. 1(a)).

Let us now consider some concrete energy spectra of the graphene Fibonacci SL modulated by the velocity barriers. Figure 2 shows the trace map for the initial Fibonacci generations of the SL in which the quasi-periodic modulation is created due to different values of the velocity barriers, namely $v_{a}=1, v_{b}=2, d=10 \mathrm{~nm}, w=5 \mathrm{~nm}$, the energy range is selected to be the minimal period equal to $2 \pi \mathrm{eV}$. The trace map investigated is characterized by the following features. For the taken set of parameters which corresponds to the trace map in Fig. 2 each Fibonacci generation forms spectra with a regular arrangement of the energy bands, and each of them exposes its own geometry. The higher generation is, the spectra of more complex pat- 


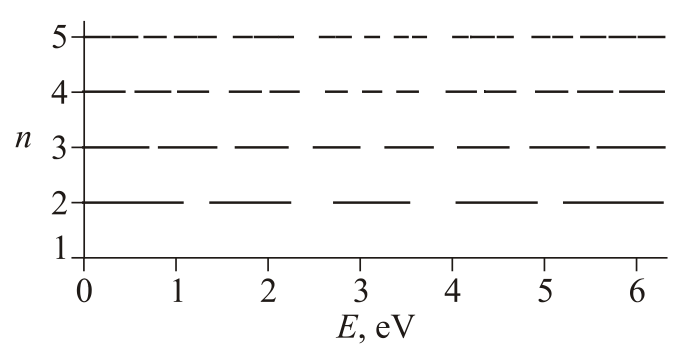

Fig. 2. Trace map for the initial Fibonacci generations, values of the parameters are as follows: $d=10 \mathrm{~nm}, w=5 \mathrm{~nm}, v_{a}=1, v_{b}=2$.

tern correspond to it. Note that spectra of higher generations are strongly fragmented (therefore we don't represent them), and besides fragmentation degree increases significantly with increase in geometrical SL parameters $d, w$.

With increasing the number of the Fibonacci sequence the number of gaps increases and their total width becomes larger. The fragmentation of the allowed bands in all generations starting from the third one occurs in accordance with the property of the self-similarity. Note also that, for some energy ranges, there are gaps in every Fibonacci sequence.

It should be noted further that in certain fixed energy areas, the Fibonacci inflation rule is satisfied: $z_{n}=z_{n-1}+z_{n-2}$, where $z_{n}$ is number of bands in the $n$th Fibonacci generation. The minimal such energy range is shown in Fig. 2. The numbers of the allowed bands in the consequent Fibonacci generations for the parameters chosen are 5, 8, 13, 21 for the 2d, 3d, 4th and 5th sequences, respectively.

The main conclusion from the spectra presented is as follows: Fibonacci quasi-periodic modulation in graphene superlattices with the velocity barriers can be effectively realized by virtue of a difference in $v_{a}$ and $v_{b}$ values, i.e. in value of the velocity barriers (no additional factor is needed). And this fact is true for a case of normal incidence of quasi-electrons on a lattice. (Therefore, the statement of authors of [14] that in graphene-based SL (in contrast to other SL), the quasi-periodic modulation can be "manifested only at oblique incidence" of the Weyl-Dirac electrons on a lattice isn't correct. As the results of this work demonstrate (and also the results of the previous works $[13,15,16])$ the implementation of the quasi-periodic modulation depends on a quasi-periodicity factor, and we see that if this factor is realized either due to different magnitude of the velocity barriers (as in this work), or by virtue of different values of gaps (as in $[15,16]$ ), the quasiperiodic modulation takes place not only at inclined incidence of quasi-particles on a lattice but also at their normal incidence.)

We have shown above that the Fibonacci quasi-periodic modulation in the graphene SL can be created due to different Fermi velocity values in the SL barriers. There is another way to form an effective quasi-periodic modulation in the SL under consideration and it is due to different values of the electrostatic barriers in different elements of the array while maintaining the velocity the same along the lattice chain. The external electrostatic potential $U$ has a significant impact on the electron transmission and it is convenient to tune the transmission spectra with the help of this potential. Let us first consider briefly the effect of the external potential $U$ on the strictly periodic SL with the velocity barriers. Denote the potential in elements $a$ and $b$ as $U_{a}$ and $U_{b}$, respectively; $U_{a}=U_{b}$ for the strictly periodic SL. The potential barriers are considered to be the piece wise constant, they are located along the SL chain ( $0 x$ axis). The changes in the transmission spectra caused by the electrostatic potential are illustrated in Fig. 3 and are as follows: 1) a new (additional) gap appears between the two adjacent gaps which exist in the case of $U=0 ; 2$ ) a shift of all gaps is observed and it depends on the value of $U$; 3 ) the gap width depends on $U$ also.

These changes are governed by the important property of the spectra - they are periodic with the potential $U$. For example, for the parameters of Fig. 3, spectra return to their initial state at intervals $\delta U=0.25 n \mathrm{eV}, n$ is an integer, i.e., the additional gap due to the external potential $U$ doesn't appear. This means that for certain values of $U$ the electrostatic barriers are perfectly transparent for the Dirac-Weyl quasi-electrons and thus there is a kind of the Klein paradox manifestation in the SL under consideration. (If $v_{a}=v_{b}=1$ we have $T(E)=1$ for all energies and values of $U$ due to the Klein tunneling.) The widening of gaps is accompanied by the narrowing of those gaps which relate to the SL with the velocity barriers for $U=0$.

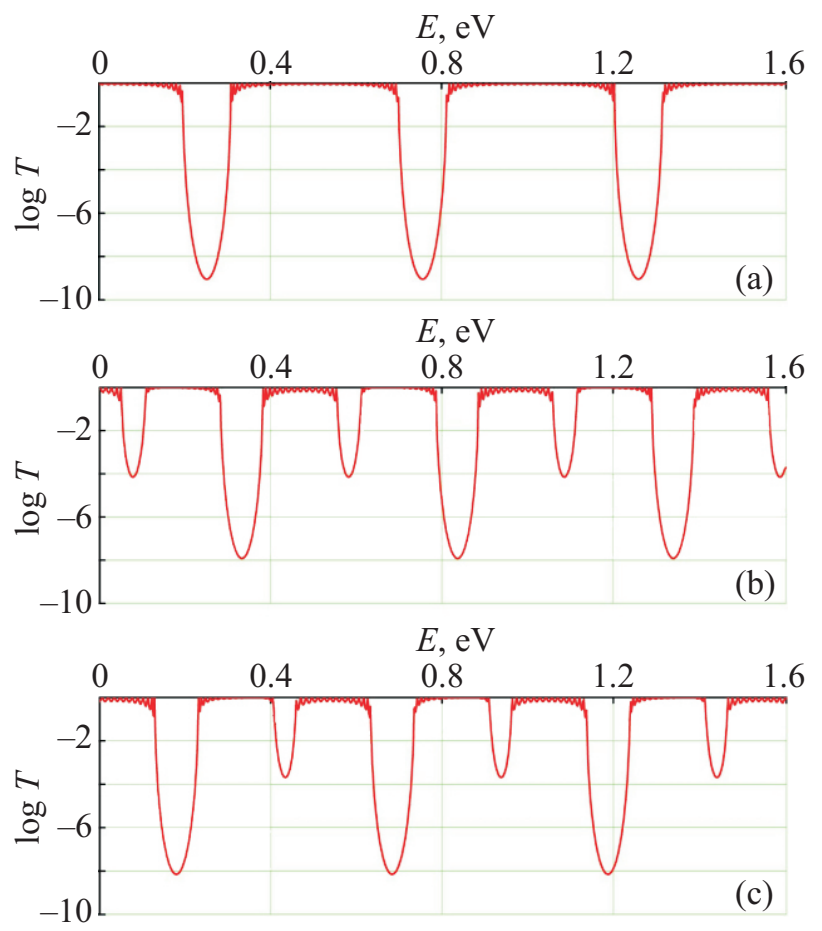

Fig. 3. Transmission spectra for the various values of the electrostatic potential $U$, eV: 0 (a), 0.16 (b), 0.36 (c); the other parameters: $v_{a}=v_{b}=2, d=10 \mathrm{~nm}, w=5 \mathrm{~nm}$. 
The magnitude of the period oscillations can be found from the following considerations. According to the Bloch theorem we can write

$$
\cos [\beta(d+w)]=1 / 2 \operatorname{Tr}\left(M_{w} M_{a}\right),
$$

$d+w$ is the lattice period. Calculation of the right side of this equation for the case of normal incidence of electrons yields the expression

$$
\cos [\beta(d+w)]=\cos [(E-U) d / v \pm E w],
$$

$\mathrm{v}=\mathrm{v}_{a}=\mathrm{v}_{b}$.

The last formula yields a value for the period of oscillations in the transmission spectra

$$
\delta U=n \pi \mathrm{v} / d .
$$

This expression determines the dependence of the period $\delta U$ on the SL geometric parameters (it is inversely proportional to the barrier width and holds for each value of the quantum well width) and on the Fermi velocity. Note that formula (10) holds well even for a small number of the SL periods.

Figure 4 shows a trace map for the SL under consideration for the difference $\Delta U=U_{a}-U_{b}=0.08 \pi \mathrm{eV}$, other parameters as in Fig. 3, the energy interval is chosen to be equal to the minimal period in Fig. 4. In general, its character is similar to that plotted in Fig. 2 but some of its features must be noted here. This trace map is regular and gaps are wider than for other values of $\Delta U$ even if they are larger than that is if the quasi-periodic factor is stronger. This is due to the fact that the spectra for the Fibonacci SL considered preserve the property of the periodicity in the case of $U_{a} \neq U_{b}$ and the factor of the quasi-periodicity is the secondary to the main property of periodicity. For values of $\Delta U=0.5 n \mathrm{eV}$ the quasi-periodicity doesn't manifest itself at all and spectra repeat the initial state, i.e., the one for $U=0$. The greatest splitting of the allowed bands is observed for values of $\Delta U$ slightly higher than $n$. The trace map is not regular and symmetric for the arbitrary parameter values (for the general case when $U \neq 0.25 n \mathrm{eV}$ ).

We see that the trace map in Fig. 4 is divided into two parts by the gap for energy equal to a little more than $0.8 \mathrm{eV}$ (for $\Delta U$ chosen). The number of bands is subjected

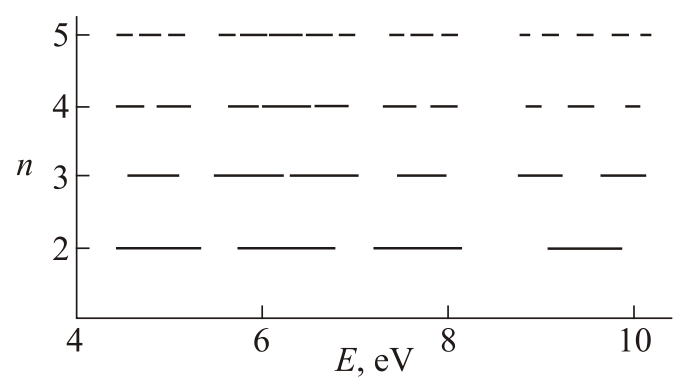

Fig. 4. Trace map for the initial Fibonacci generations of the SL with the parameters: $U_{a}=0, U_{b}=0.25 \mathrm{eV}, v_{a}=v_{b}=2, d=$ $=10 \mathrm{~nm}, w=5 \mathrm{~nm}$. to the Fibonacci inflation rule in every part: for the initial Fibonacci generations we have the sequence of numbers 3 , $4,7,11 \ldots$ and $1,2,3,5 \ldots$ in the left and right parts, respectively, and totally $4,6,10,16 \ldots$ which differs from the case of Fig. 2.

Pay particular attention to the broad (lower energy) bands in each Fibonacci generation in Fig. 4. They correspond to the so-called additional or superlattice Dirac bands in a periodic lattice [22]. It plays an important role in the controlling of the SL energy spectra since it is robust against the structural disorder. The location of the middle of such a band (mid-gap) $E_{D}$ is determined by the condition [22]

$$
q_{d} d+q_{w} w=0
$$

which yields

$$
E_{D}=U d /(d+v w) .
$$

This equation for the position of the Dirac superlattice gap is well satisfied for a wide range of the parameters involved even for a small number of the SL periods. The Dirac band width depends on the problem parameters and may be less than the width of the other (Bragg) bands (see, e.g., $[15,16,18])$.

Similar Dirac superlattice gaps exist also in the case of the quasi-periodic Fibonacci SL investigated. The mid-gap position of such a gap may be approximately found by the Eq. (13) (for not a large difference between $U_{a}$ and $U_{b}$ ). Note further that a characteristic feature of the SL Dirac band is that it doesn't depend on the lattice period $d+w$, but it is sensitive to the ratio $w / d$. This is illustrated in Fig. 5 where $\log T(E)$ is plotted for the fourth Fibonacci generation with the parameters: $v=2, U_{a}=0.32 \mathrm{eV}, U_{b}=$ $=0.28 \mathrm{eV}$, the dashed line in Fig. 5(a) corresponds to values $d=8 \mathrm{~nm}, w=6 \mathrm{~nm}$, for the solid line $d=9.6 \mathrm{~nm}, w=7.2 \mathrm{~nm}$; for the solid line in Fig. 5(b) $d=6 \mathrm{~nm}, w=8 \mathrm{~nm}$, for the dashed line $d=8 \mathrm{~nm}, w=6 \mathrm{~nm}$.

We have calculated also the values for the structure considered that can be measured in practice namely the conductance and the Fano factor using the known formulae (it is convenient to use the dimensionless conductance, see, e.g., [22] and references therein):

$$
\begin{gathered}
G^{\prime}=\int_{0}^{\pi / 2} T\left(E, k_{y}\right) \cos \theta d \theta, \\
F=\frac{\int_{-\pi / 2}^{\pi / 2} T(1-T) \cos \theta d \theta}{\int_{-\pi / 2}^{\pi / 2} T \cos \theta d \theta} .
\end{gathered}
$$

Values of $G^{\prime}(E)$ and $F(E)$ depends in general on the geometric parameters of the superlattice, on the number of SL periods, on the external potential and on the Fermi velocity 

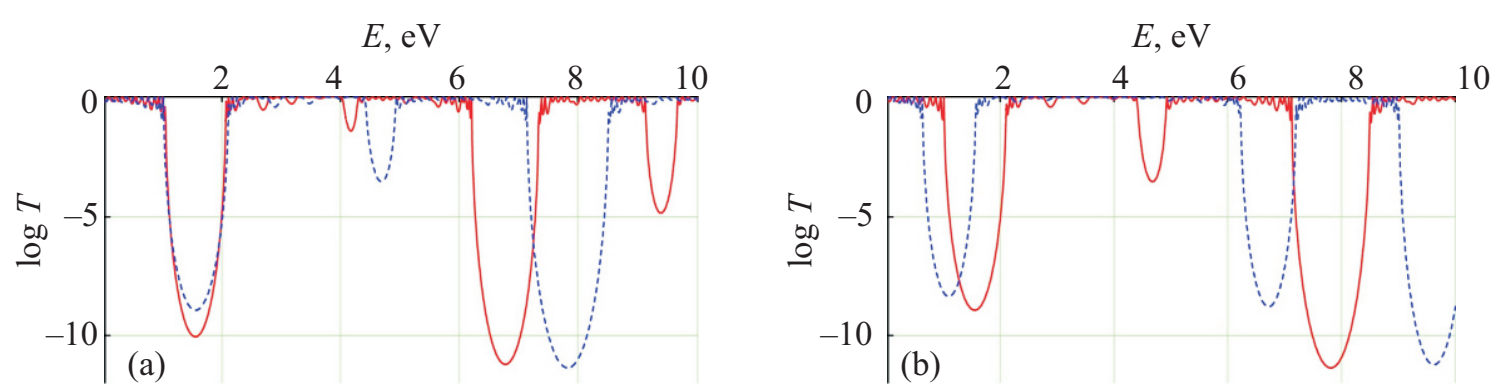

Fig. 5. (Color online) Dependences of $\log T$ on energy $E$ for the fourth Fibonacci generation, values of the parameters: $v=2, U_{a}=$ $=0.32 \mathrm{eV}, U_{b}=0.28 \mathrm{eV}$, the solid line in (a) corresponds to values $d=9.6 \mathrm{~nm}, w=7.2 \mathrm{~nm}$, for the dashed line $d=8 \mathrm{~nm}, w=6 \mathrm{~nm}$, for the solid line in (b) $d=6 \mathrm{~nm}, w=8 \mathrm{~nm}$, for the dashed line $d=8 \mathrm{~nm}, w=6 \mathrm{~nm}$.

magnitude in different elements of the lattice. The dependence of $G^{\prime}$ and $F$ on the quasi-electron energy $E$ is presented in Figs. 6, 7 for the strictly periodic superlattice with the parameters: $d=w=10 \mathrm{~nm}, U_{a}=U_{b}=0$, the number of the periods $n=8, v_{b}=2,3,4$ for the curves in Figs. 6(a), 6(b), 6(c) and 7(a), 7(b), 7(c), respectively.

We see that the dependences of $G^{\prime}$ and $F$ on $E$ are indeed sensitive to the Fermi velocity value; $G^{\prime}(E)$ significantly decreases with increasing of $v_{b}$ and the Fano factor deviates substantially from the universal value of $1 / 3$ for the most values of the parameters involved being close to unity in those areas of energy which correspond to wide gaps in the $T(E)$ dependence. Note also that we chose the values of the parameters so that the minimum number of the minima in the $G^{\prime}(E)$ dependence (in one period) is exactly equal to the Fermi velocity values in the barrier regions, namely $v_{b}=2,3,4$ for the curves in Figs. 6(a), 6(b), 6(c), respectively; the size of one period becomes larger with the Fermi velocity increasing and it is equal to $2 \pi, 3 \pi$, $4 \pi$ in Figs. 6(a), 6(b), 6(c), respectively (the periodicity of the spectra for the graphene structures with the velocity barriers was analyzed in detail in $[19,20])$.
Values of the Fermi velocity in Figs. 7(a), 7(b), 7(c) are equal to $v_{F}=2,3,4$, respectively.

Figure 8 shows the spectra for $G^{\prime}(E)$ and $F(E)$ for the SL containing the fourth Fibonacci generation with the parameters: $d=w=10 \mathrm{~nm}, U_{a}=U_{b}=0, v_{b}=2$. We used only 2 superlattice periods and, interestingly, this is enough for realization of the efficient quasi-periodic modulation. The minima in the conductivity are associated with the maxima in the Fano factor dependence on $E$.

The calculations show that there are regions in the $G^{\prime}(E)$ and $F(E)$ dependences which correspond to the Dirac superlattice gaps. These energy areas do not change their position as the value of the lattice period $d+w$ changes, while the other extremes are shifted on the energy axis. At the same time, the Dirac gap position is sensitive to the ratio $d / w$. This is evident from Fig. 9 for the SL for the fourth Fibonacci generation with the parameters: $d=9.6 \mathrm{~nm}$, $w=7.2 \mathrm{~nm}$ for Figs. 9(a), 9(c) and $d=8 \mathrm{~nm}, w=6 \mathrm{~nm}$ for Figs. 9(b), 9(d), other parameters are as follows: $v_{F}=2$, $U_{a}=0.32 \mathrm{eV}, U_{b}=0.28 \mathrm{eV}$.
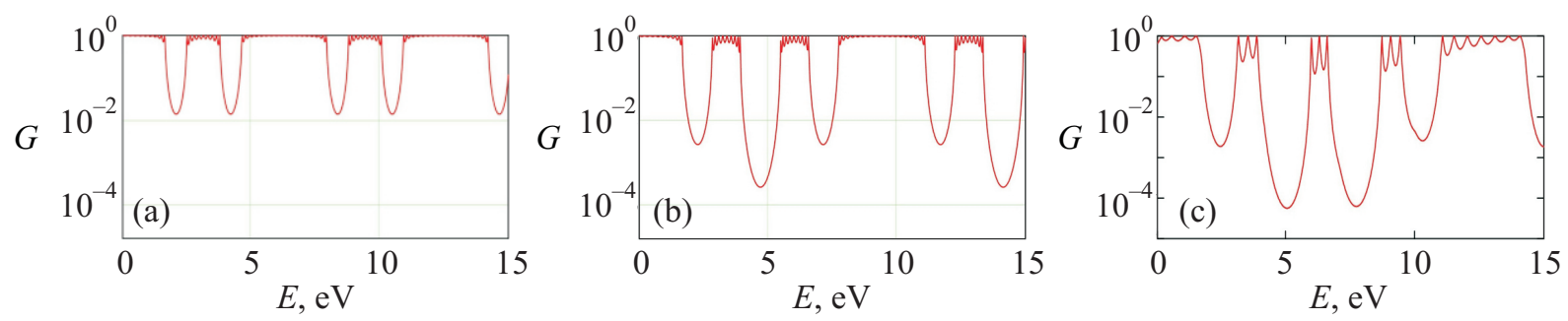

Fig. 6. Dependences of the conductance on energy for the strictly periodic SL with different Fermi velocity values $v_{b}=2$ (a), 3 (b), 4 (c).
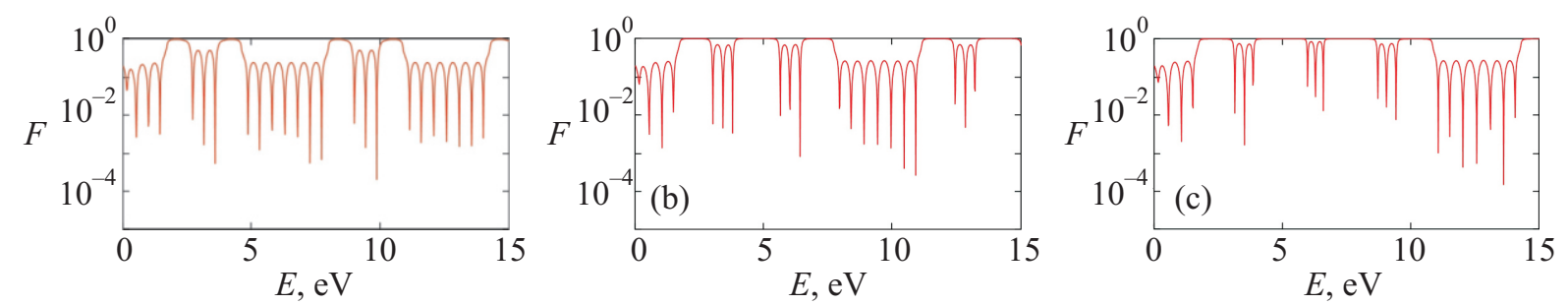

Fig. 7. Fano factor versus energy for the strictly periodic SL with different Fermi velocity values. 

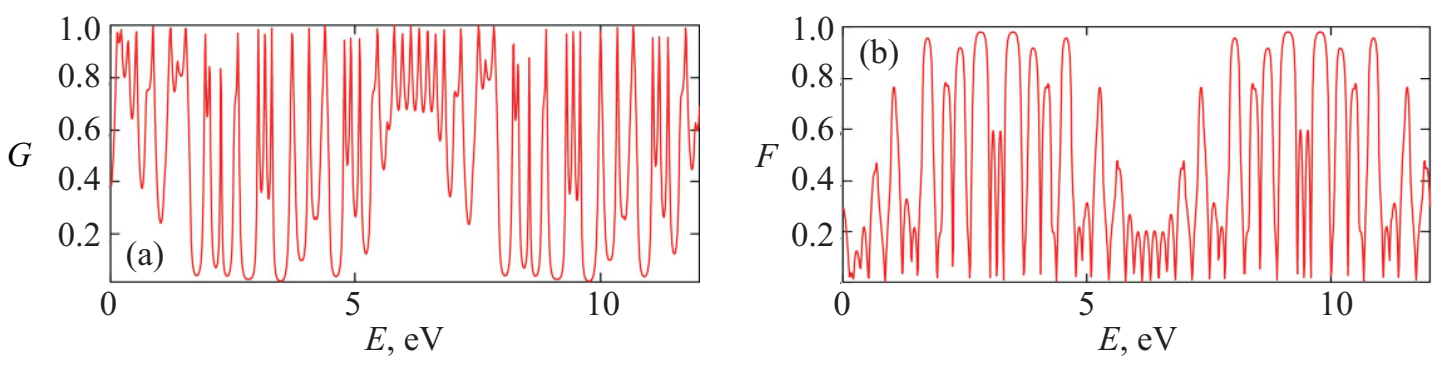

Fig. 8. Dependences of the conductance (a) and the Fano factor (b) on energy for the SL related to the fourth Fibonacci generation.
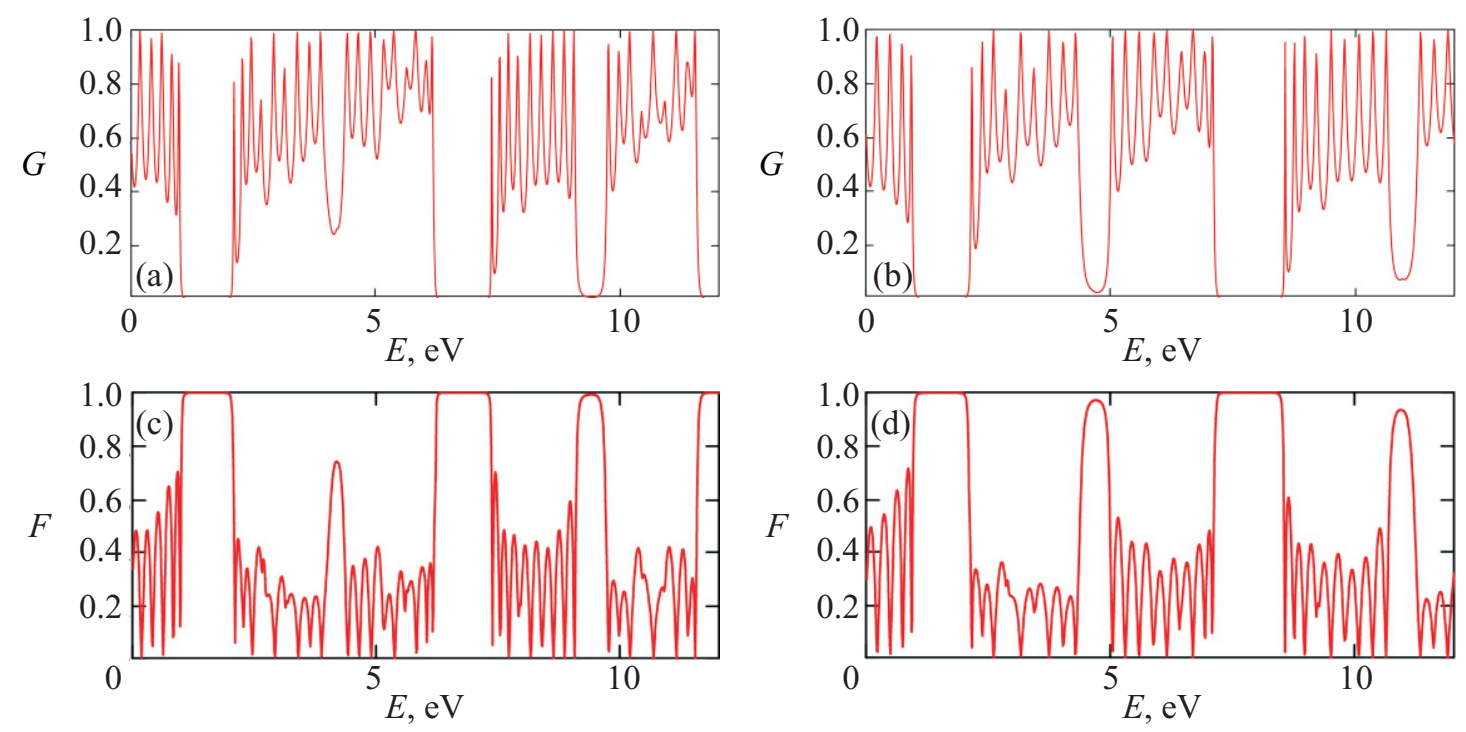

Fig. 9. Dependences of the conductance (a), (b) and the Fano factor (c), (d) for the SL for the fourth Fibonacci sequence with different values of $U, d, w$.

\section{Conclusions}

We analyze the transmission spectra of the Fibonacci superlattice based on graphene modulated by the Fermi velocity barriers. The dependences of the transmission rates, of the conductance and of the Fano factor on the quasielectron energy are calculated and analyzed. The quasiperiodic modulation can be realized due to different values of the velocity barriers or due to different values of the external potential in the SL elements $a$ and $b$. Contrary to the case of other types of the graphene SL spectra studied reveal the periodic character over all the energy scale and the transmission coefficient doesn't tend asymptotically to unity at rather large energies. The periodic dependence of the considered spectra on the magnitude of the external electrostatic potential is observed the period being proportional to the quantity $n \pi$ ( $n$ is an integer) and inversely proportional to the barrier width. Spectra demonstrate the rich variety of configurations (patterns) of the allowed and forbidden bands location dependent on one hand on the Fermi velocity magnitude and on the other hand on the SL geometry; for some special parameter values, they expose the regular character, symmetrical with respect to a certain point. Spectra of higher generations are strongly fragment- ed and besides fragmentation degree increases significantly with increase in geometrical SL parameters $d, w$ (the width of the barrier and of the quantum well, respectively). The higher generation is, the spectra of more complex pattern correspond to it. In the certain fixed energy areas the spectra are subjected to the Fibonacci inflation rule: $z_{n}=z_{n-1}+z_{n-2}$, where $z_{n}$ is the number of bands in the $n$th Fibonacci generation. There is another way to form an effective quasiperiodic modulation in the SL under consideration and it is due to different values of the electrostatic barriers in different elements of the array while maintaining the velocity the same along the lattice chain. The dependence of the conductance on energy reveals the periodical character and, in particular, one can choose the values of the parameters so that the minimum number of the minima in the $G^{\prime}(E)$ dependence (in one period) is exactly equal to the Fermi velocity values in the barrier regions. The SL Dirac gaps are present in the spectra and their location depends on the velocity barriers value, on the value of the external potential as well as on the SL geometrical parameters. The results of our work can be applied for controlling the energy spectra of the graphene-based devices.

It's a pleasure for us to express our gratitude to Dr. S.I. Litvynchuk for the technical assistance. 
1. A. Geim and K. Novoselov, Nat. Mater. 6, 183 (2007).

2. A.N. Castro Neto, F. Guinea, N.M.R. Peres, K.S. Novoselov, and A.K. Geim, Rev. Mod. Phys. 81, 109 (2009).

3. J.M. Pereira, F.M. Peeters, A. Chaves, M. Barbier, and P. Vasilopoulos, Semicond. Sci. Techn. 25, 033002 (2010).

4. V.V. Cheianov and V.I. Falko, Phys. Rev. B 74, 041403 (2006).

5. L. Tapaszto, G. Dobrik, P. Nemes-Incze, G. Vertesy, Ph. Lambin, and L.P. Biro, Phys. Rev. B 78, 33407 (2008).

6. A. Raoux, M. Polini, R. Asgari, A.R. Hamilton, R. Fazio, and A.H. MacDonald, Phys. Rev. B 81, 073407 (2010).

7. P.M. Krstajic and P. Vasilopoulos, J. Phys.: Condens. Matter 23, 135302 (2011).

8. Liu Lei, Li Yu-Xian, and Liu Jian-Jun, Phys. Lett. A 376, 3342 (2012).

9. A. Concha and Z. Tesanovic, Phys. Rev. B 82, 033413 (2010).

10. Y. Wang, Y. Liu, and B. Wang, Physica E 53, 186 (2013).

11. P. Zhao and X. Chen, Appl. Phys. Lett. 99, 182108 (2011).

12. Lu Wei-Tao, Wang Shun-Jin, Wang Jong-Long, Jiang Hua, and Li Wen, Phys. Lett. A 377, 1368 (2013).

13. Xu Yafang, Zou Jianfei, and Jin Guojun, J. Phys.: Condens. Matter 25, 245301 (2013).

14. A.N. Korol and V.N. Isai, Phys. Solid State 55, 2596 (2013).

15. А.Н. Король, ФНТ 40, 324 (2014) [Low Temp. Phys. 40, 251 (2014)].

16. Z. Cheng, R. Savit, and R. Merlin, Phys. Rev. B 37, 4375 (1988).

17. A.N. Korol and V.N. Isai, Springer Proceegings in Physics 156, 43 (2014).

18. P.V. Ratnikov and A.P. Silin, JETP Lett. 100, 311 (2014).

19. L.-F. Sun, C. Fang, and T.-X. Liang, Chin. Phys. Lett. 30, 047201 (2013).

20. L.-G. Wang and X. Chen, J. Appl. Phys. 109, 033710 (2011).

21. Neetu Agrawal (Garg), Sameer Grover, Sankalpa Ghosh, and Manish Sharma, J. Phys.: Condens. Matter 24, 175003 (2012).

22. Jonas R.F. Lima, Luiz Felipe C. Pereira, and C.G. Bezerra, J. Appl. Phys. 119, 244301 (2016).

\section{Енергетичні спектри квазі-періодичної надгратки на основі графрену}

\section{А.М. Король, А.І. Соколенко, І.В. Соколенко}

В континуальній моделі методом трансферних матриць розраховано та проаналізовано спектри трансмісії діраківських квазіелектронів крізь квазіперіодичну надгратку (НГ) Фібоначчі. Розглядається одновимірна НГ на основі моношарового графену, модульована бар'єрами швидкості Фермі. Запропоновано використати новий квазіперіодичний фактор. Показано, що квазіперіодична модуляція Фібоначчі в графенових надгратках із бар'єрами швидкості Фермі може бути ефективно реалізована завдяки різниці в значеннях бар'єрів цієї швидкості (додатковий фактор не потрібен, i слід зазначити, що не кожен фактор може забезпечити квазіперіодичну модуляцію). Цей факт справедливий для випадку нормального падіння квазіелектронів на гратку. На відміну від інших типів вивчених спектрів трансмісії в графенових НГ в даному випадку виявляється нетривіальна їх властивість — періодичність по всій шкалі енергії, так що коефіцієнт пропускання не наближається асимптотично до одиниці при достатньо високих енергіях. Розраховано та проаналізовано провідність (з використанням відомої формули Ландауера-Буттікера) та фактор Фано для даної структури. Проаналізовано залежність спектрів від величини швидкості Фермі та від зовнішнього електростатичного потенціалу, а також від геометричних параметрів НГ (ширин бар'єрів і квантових ям). Використовуючи розглянуті квазіперіодичні НГ, можна регулювати транспортні властивості графенових структур в широкому діапазоні іх параметрів. Отримані результати можуть бути використані для застосування в електроніці на основі графену.

Ключові слова: графен, надгратка Фібоначчі, бар'єри швидкості Фермі, спектри трансмісії. 